\title{
Research on transmission of technology of downward information security for wellbore trajectory control
}

\author{
Lin DeShu ${ }^{1}$ and Feng Ding ${ }^{2^{*}}$
}

\begin{abstract}
Information transmission is an important part of the wellbore trajectory automatic control system. Combined with the characteristics of the guided drilling system, drilling fluid pulse is selected as a tool for trajectory control. Negative pulse transmission of drilling fluid is one of the key technologies to realize wellbore trajectory automatic control. In this paper, the working principle of negative pulse propagation of drilling fluid is introduced. The characteristics of negative pulse propagation and signal pretreatment of drilling fluid are analyzed emphatically. Signal correlation analysis method is proposed for signal transmission of drilling fluid negative impulse well. In this paper, the propagation characteristics of drilling fluid pressure pulsed downward transmission system are studied deeply. The influence of various drilling parameters on the drilling fluid pressure pulse is analyzed, and the model of drilling fluid pressure pulse is presented. Experimental results show that the proposed method can effectively separate useful signals from interfering signals. It is verified by field experiment that the success rate of information decode system designed by this method is high, which can meet the requirement of well trajectory precise control.
\end{abstract}

Keywords: Drilling fluid negative pulse, Wellbore trajectory control tool, Downward information, Propagation characteristics

\section{Introduction}

Wellbore trajectory control closed-loop drilling technology is the late 20th century developed advanced automated drilling technology. It combines computer control technology in drilling tools, representing the world's highest level of development of drilling technology. The world's drilling technology has undergone a qualitative leap. The outstanding characteristic of the system is that the monitoring and control work is completed by the microcomputer-controlled intelligent actuator in the underground, and the ground can also be remote controlled. Under the condition of no ground personnel intervention, the closed-loop drilling control system automatically tracks along the well-designed 3D well trajectory. The actual drilling conditions and the status of the underground tool can be sent to the ground in real time by the mud pulse generator, which was monitored

\footnotetext{
* Correspondence: tea027@126.com

${ }^{2}$ School of Mechanical Engineering, Yangtze University, Jingzhou, Hubei 434023, China

Full list of author information is available at the end of the article
}

by field operators. When there is an abnormal situation in the ground, the ground remote control and intervention instructions will be sent to the underground tools. By changing the working parameters of the controller in the drilling tool, the drill bit attitude can be changed and the well trajectory can be controlled.

Usually, "Uplink" is used to represent the communication from underground to the ground, with "Downlink" from ground to underground communication. "Uplink" and "Downlink" form the two-way communication technology of ground and underground [1]. To achieve a wellbore trajectory control, one must use the underground real-time control or ground that can be remoted by a well trajectory control tool. The tool can measure the real-time position of the drill bit and realizes realtime control of the underground. In wellbore trajectory control drilling, the wellbore trajectory control tool is the core of wellbore trajectory control drilling system. It is also fundamental to achieve a wellbore trajectory control. Underground real-time control system or ground monitoring system, according to the design of the well 
trajectory and measurement information, can real-time monitor the underground tools and remote control. Measurement and transmission system is as much as possible near the drill bit that measured the spatial attitude of the borehole and other information, and promptly sent to the underground computer and the ground computer for signal processing. The ground monitoring system through the "Downlink" issued new control instructions, the well trajectory control tool in accordance with the control command to operate, in order to achieve well trajectory track control. In the process of realizing the closed-loop drilling system of borehole trajectory control, it is necessary for the ground to interfere with the working posture of underground tools. Ground intervention control is a necessary means to realize wellbore trajectory control in closed-loop drilling system. Therefore, the ground-to-underground remote control means and the ground to the underground data transmission method has become a hot industry issues related to the study. The signal transmission in the wellbore is a bridge connecting the ground monitoring system with the underground tracking system and is also the key technique to realize the closed-loop control of the wellbore trajectory. Ground-to-underground information transmission is directly related to the working state of underground tools. Accurate and efficient "Downlink" technology can improve drilling efficiency. Therefore, the ground-to-underground signal transmission system, the "Downlink" technology research, is very important.

\section{Related work}

A variety of methods have been used to transmit signals from the ground to the equipment in the bottom hole assembly. At 90 years, some scientists have proposed to carry out underground operations by controlling the change in the rotational rate of the drill pipe. Using the drill pipe rotation rate as the basis for the transfer command, the sensor connected to the drill pipe can measure the rotation rate of the drill pipe. The rotation axis of the sensor is coincident with the rotation axis of the drill pipe. When the rotational speed increases, the centrifugal force increases, in the role of centrifugal force sensor in the radial extension. Whereas a decrease in the rate of rotation of the drill pipe will cause the sensor to retract, that is, the drill pipe rotation rate may be indirectly expressed as a radial expansion of the sensor. Using a sensor, a spring, and an excitation source, the instrument can perform command transfer based on drill string rotation rate changes. The mechanical structure of the entire sensor and excitation system is very complex and can only measure the change in the rate of rotation of the drill pipe. It cannot measure the increase in the angle of rotation of the drill pipe. This method may cause large errors in the signal transmission when the drill string is rotated at a low rate due to the centrifugal force acting on the sensor in drilling the inclined shaft.

Mougel et al. proposed that the information is conveyed by one or more different rotational angle codes. In the drilling, a discontinuous angular displacement is detected by an underground gyroscope and the command is solved in the microprocessor. The intermittent command codes are generated by successive rotations of the drill rod at different angular velocities, which are also detected by the gyroscope and transmitted to the microprocessor for decoding. The decoded command is then sent to the control tool by the microprocessor to complete the delivery of the command [1]. In 2001, Schlumberger invented two kinds of signal transmission methods; one is the drill rod to rotate the command code generated by the continuous different angular speed and the detection of underground rotary instrument. The downlink command transmission system decodes in the microcomputer. The other is the use of underground rotary tool-sensitive drill pipe rotation angular velocity to generate the command code. In the microcomputer, the system decodes and sends the decoded command to control the underground equipment. The invention provided a comparatively economical transmission system, which avoided costly devices, and its downward transmission system was relatively complete. The system had an error detection function and the fault detection system was not affected by formation factors. In the reference [2], it was proposed by using inclinometer and three-axis magnetometer to monitor the rotation angle of drill pipe rotation rate, through the underground equipment to deal with inclinometer and three-axis magnetometer output command code. The magnetometer output in this method is affected by formation parameters and can only be used in uncased hole. These inventions provided a downstream command transmission method without a mud circulation system.

In 1999, Gruenhagen invented the use of mud as a medium that will be sent to the underground command, to complete the pressure pulse modulation in the ground, the pressure pulse in the underground by the pressure or flow sensor sensitive, by the microprocessor decoding and the command sent to the controller [3]. During drilling, a constant flow rate of mud is pumped from the mud pool with a main pump. Most of the mud into the mine, to maintain the mud circulation, bypass valve equipment controlled by the electronic controller. When the valve was opened and closed, a flow pulse was generated. The amplitude and time of such a pulse could be controlled. The speed of these pulses can be detected by suitable underground equipment, such as flow meters, pressure gauges, and turbines. Data transmission is 
based on binary modulation pulse. In 2005, Halliburton, as the representative of the downlink channel remote transmission system invented by Finke et al., provides improved equipment and method for remote command transmission. By the pressure pulse from the ground equipment, it sent instructions to the bottom hole assembly [4]. The utility model is composed of a ground transmitter which generates a pressure pulse signal, a control system, and an underground receiver which is used for receiving and decoding the pulse signal. When the bypass valve was opened and closed at work, a series of pressure pulse signals which could be received and decoded by the underground receiver could be generated. This method reduced the time spent in the downlink channel transmission without interrupting the drilling. If the upward and downward channel signals were transmitted at different frequencies, the bidirectional transmission could be realized. The remote transmission scheme and software program provided an innovative method for the filtering and decoding of downlink channel signals. The software program determined the time interval between the peak values of the pulse and decoded them into commands. The software program also included an error check that was completely received. The utility model was characterized in that the utility model related to a pressure pulse which was generated by the ground equipment to transmit the instruction to the down hole drill assembly without interrupting the drilling. The pressure pulse signal was detected using a down hole receiver, and the pressure pulse signal was decoded by a software program. It could also achieve a two-way communication between the ground equipment and the bottom hole assembly at a relatively fast transfer rate; so, this method was particularly practical.

European Patent GB2412128 implemented as follows [5]: The underground tool was equipped with a mudpowered engine and a sensor to monitor the rotor speed, using the electrical signal from the sensor as the command signal for the downstream channel. In the process of using this method, the bit should be lifted to avoid the friction between the drill bit and the bottom hole assembly. Downstream signals could be encoded by the frequency, amplitude, or speed of mud flow changes. The underground unit detected changes in the bottom hole assembly speed below the mud power engine for decoding purposes, with different speeds representing different commands. Patent US2006225920 in the downlink channel system is the use of mud flow rate changes, sending ground command signal into the down hole control drilling tool combination. First, the signals from the ground were transmitted by varying the flow into the wellbore, and the downstream signals could be sent using a deterministic or dynamic time-table. Second, the command might be sent based on the number of times the flow exceeds a certain threshold; then, the down command might be defined by a period of constant flow rate. The commands corresponding to each determination signal were stored in an underground reservoir, and the mud flow rate on the ground was automatically controlled by a controller or mud flow rate control device that controlled the mud pump. Changes of mud flow rates could be monitored by system-matched detectors. In addition, Herrmann was used to communicate with underground tools and instruments, and the response of the underground device to the transmitted coded information was dependent on the change of pressure during communication [6].

All in all, the general method for transmitting signals from downstream channels is through mud pulse telemetry. When drilling, the mud is pumped underground and the underground receiver at the bottom hole assembly can measure pressure and mud flow. Mud pulse telemetry is a method of transmitting a series of transiently varying pressure or pulse signals. In the drilling fluid, the signal can be detected by the receiver. As a result of the downstream channel signal transmission, the underground receiver can detect the pressure pulse, which includes the pulse width, pulse width, and pulse interval, and then decoded into a detailed instruction to the actuator. The idea of transmitting signals from the ground to the underground equipment through a mud pulse telemetry system has been put into practice in the past. The most common method of generating a pressure pulse signal is by interrupting the drilling and continuously switching the mud pump at a fixed frequency. The pressure pulse is transferred from the mud to the bottom of the well to control the underground actuator [7].

The paper mainly deals with the transmission characteristics of the mud pulse signal in the wellbore [8], velocity research [9-11], and so on. According to the proposed negative pulse channel slurry program [12], the signal transmission mode was studied. Xi'an Petroleum Exploration Equipment General Factory Zhang Wei and other geological transmission system of information transmission channel, a positive pulse generator system for further study of geological orientation, laid a good foundation [13]. China Petroleum Exploration and Research Institute is drilling Li Lin, who has used the wireless short-pass method of geological guidance in the underground data short-distance transmission performance studied [14]. The Institute of Underground Measurement and Control of Xi'an Petroleum University put forward the signal transmission using the different vibration frequency of drill pipe static and vibration [15-17]. Development of prototype field experiments uses mud pulse width modulation method to achieve the 
downlink channel [18]. In 2004, Yang Quanjin of Shengli Petroleum Administration Bureau proposed to realize the transmission of control signals from ground to underground by controlling the penetration rate of drilling tools [19].

There are two main ways to transfer commands, that is, the use of rotary drill string to transfer signals and the use of mud circulating fluid transmission signal. Now, people on the mud pulse and rotation method of signal transmission study how to improve the quality of signal transmission and signal transmission in other ways. Correspondingly, these signals are sent down to the corresponding decoding method, so that the underground controller can correctly receive these commands. In recent years, there are two kinds of underground data processing methods: one is to identify the method of loading the signal in the drilling fluid and the system [20], which is Bayesian method to identify the ground sent to the underground signal and the other is noise detection and filtering system [21], that is, adaptive filtering method to deal with mud pulse signal.

It can be seen from the previous analysis that the current method is still in ground open-loop control. Ground intervention control is a necessary means to realize closed-hole well trajectory control drilling system. Underground information transmission is an important way of well trajectory remote control and manual intervention of trajectory automatic control. When an anomaly occurs, the ground sends an intervention command to the underground controller via the "downward transmission system," thereby changing underground tool parameters. From the ground according to a certain coding method to send the command to the underground, it can control guided drilling process. Therefore, the ground and underground information transmission channel formed by the coding transmitter and the decoder is an important part of closed-loop well trajectory control drilling research.

\section{Principles of information downward transmission system}

\subsection{Drilling site composition}

The basic structure of the transmission system of drilling guide parameters is shown in Fig. 1. The drilling fluid is more suitable for the actual situation of drilling engineering pulse as the transmission medium realizes the drilling parameters oriented downward transmission.
Drilling fluid pulse can be divided into three types: positive pulse, negative pulse, and continuous wave. In the MWD system, the positive pulse and continuous wave are generally used. Positive pulse mode is that pulse generator is arranged in the drill collar. It can instantly impede the flow of drilling fluid, resulting in higher than normal pressure of the pressure wave [22]. In the drilling guide parameter system, the negative pulse form is better. The negative impulse is the pressure wave which is lower than the normal pressure, or the method of judging the flow pulse. The overall scheme of drilling guide parameters based on negative pulse is shown in Fig. 2.

\subsection{Computer-controlled downward transmission mechanism}

The drilling efficiency of the drilling operation is seriously affected by the man-made control of the opening and braking of the ground mud pump, and there are uncertainties such as man-made error of control time and artificial judgment. This is the main obstacle to improve the speed of information transmission. A computercontrolled bypass valve is installed at a suitable location on the ground or in the well, bypassing a portion of the slurry that is involved in the normal circulation to cause a change in the down hole pressure. Down hole information can be transmitted by changing the down hole pressure, and the downward change of pressure can be realized. Moreover, computer control has a great advantage in terms of time accuracy and signal frequency. The control mechanism of computer-controlled downstream communication is still negative pulse of mud as the information carrier. The generation of mud negative pulse is no longer controlled by the operator, but the computer-controlled switch device is added to the ground to control the effective flow into the bottom mud. When the mud flow into the bottom of the well decreases in a certain period of time, this time and the former moment will appear the reduction phenomenon in the mud pressure contrast, namely has produced the negative pulse. The complete set consists of a ground launch device that generates pressure pulse signals, a control system, and a down hole receiver for receiving and decoding the pulse signal. The ground switchgear is replaced by a mud bypass valve that, during operation, opens and closes to produce a series of pressure pulse signals that can be received by the down hole receiver and decoded. It is worth noting that this method

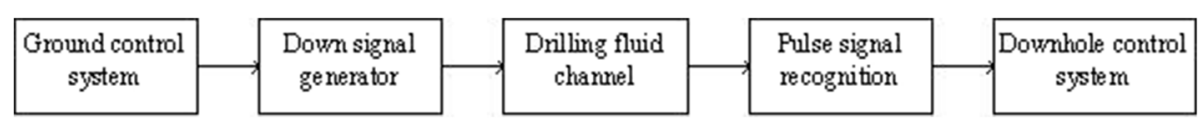

Fig. 1 The basic structure of the transmission system of drilling guide parameters 


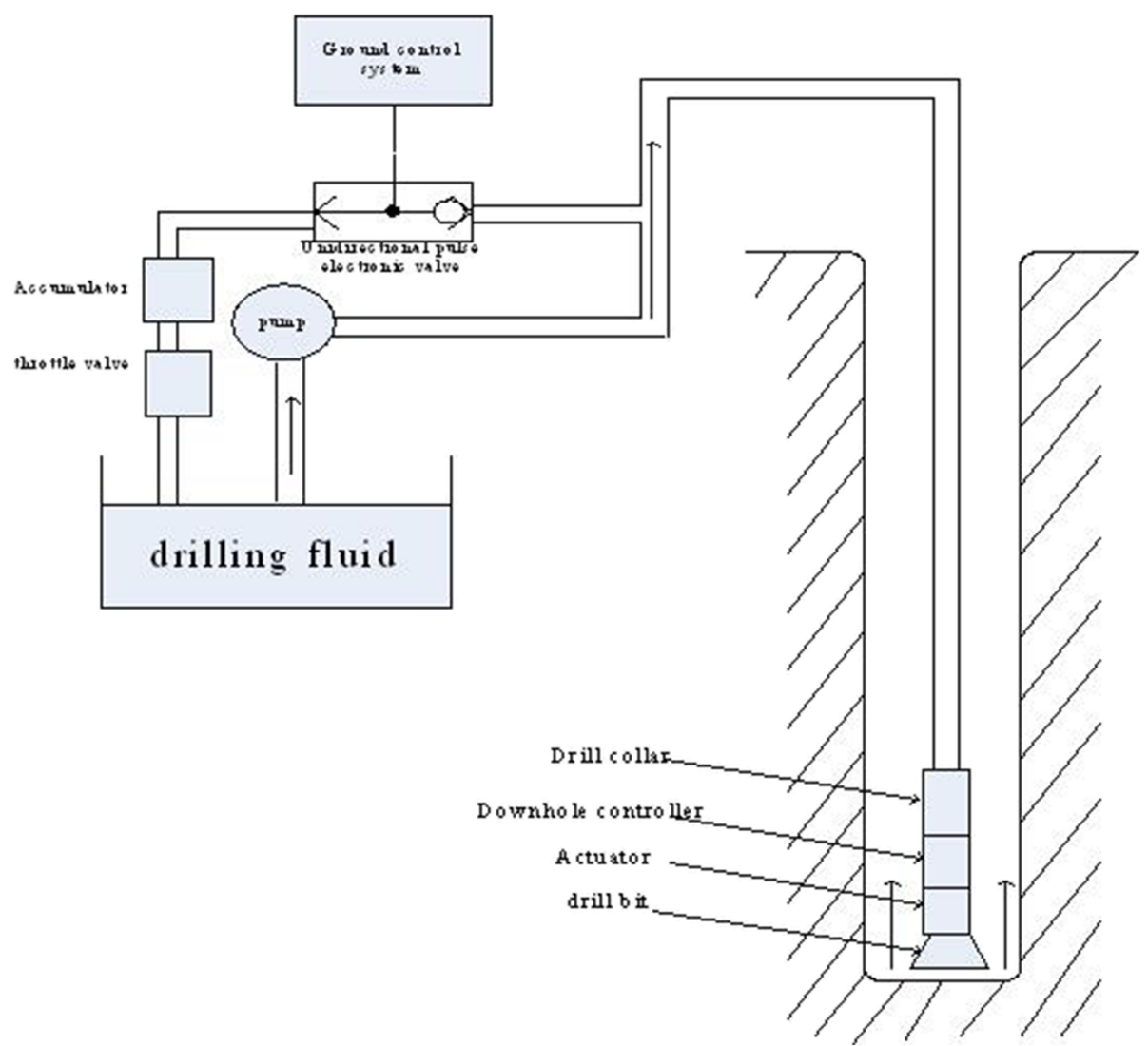

Fig. 2 Integral scheme of drilling guide parameters down transmission based on drilling fluid negative pulse

shortens the time necessary for downstream information transmission without interrupting the drilling and simultaneous uplink transmission.

\subsection{Secure encryption method for downlink information}

In the drilling, fluid pulse communication system can be used in the encryption algorithm AES (Advanced Encryption Standard), this algorithm is also known as Rijndael encryption. AES is proposed by the US National Institute of Standards and Technology (NIST) to replace DES encryption standard. AES encryption algorithm uses symmetric block cipher system, the key length of the support for the 128, 192, 256 length of 128 bits; the algorithm should be easy to achieve by a variety of hardware and software.

We can use different information security levels, with different key lengths to achieve, as shown in Table 1 [5]. Here, not only considering the classification of data security requirements, taking into account the network and the hardware implementation cost of the actual operation process, we put the wireless network data communications using the 128-bit key length, this mainly in order to save the computational overhead in mobile networks.

\subsubsection{SubBytes function}

The SubBytes function is the only non-linear mixing step of AES algorithm, the 16 bytes of each of parallel map for new bytes. The SubBytes function is the implementation shown in Fig. 3.

\subsubsection{ShiftRows function}

The ShiftRows function is to state data in each row in the rotate right $0,1,2$, and 3 bits. The state transformation is very easy to implement. The ShiftRows function implementation is shown in Fig. 4, where ROLi represents Rotate Right i bits.

Table 1 Encryption-level classification

\begin{tabular}{lll}
\hline Security level & Example & Key length \\
\hline High & $\begin{array}{l}\text { Private information, bank, } \\
\text { and confidential business information }\end{array}$ & 256 \\
Normal & Private information & 192 \\
Little & Wireless information & 128 \\
Not require & Public information & No encryption
\end{tabular}

AES algorithm accepts a 128-bit plaintext, and a 128,192, 256 for the key under the control to generate a 128-bit ciphertext. Specific operations by known round (round) a collection of steps, in which the number of rounds can be $9,11,13$ (depending on key length). AES has a four-step operation: (1) SubBytes, (2) ShiftRows, (3) MixColumns, and (4) AddRoundKey 


\begin{tabular}{|c|c|c|c|c|c|c|c|}
\hline$a_{0,0}$ & $a_{0,1}$ & $a_{0,2}$ & $a_{0,3}$ & $b_{0,0}$ & $b_{0,1}$ & $b_{0,2}$ & $b_{0,3}$ \\
\hline$a_{10}$ & $a_{1,1}$ & $a_{1,2}$ & $a_{13}$ & $b_{1,0}$ & $b_{1,1}$ & $b_{1,2}$ & $b_{13}$ \\
\hline$a_{2,0}$ & $a_{2,1}$ & $a_{2,2}$ & $a_{23}$ & $\mathrm{~b}_{2,0}$ & $\mathrm{~b}_{2,1}$ & $\mathrm{~b}_{2,2}$ & $b_{2,3}$ \\
\hline$a_{3,0}$ & $a_{3,1}$ & $a_{32}$ & $a_{33}$ & $b_{3,0}$ & $b_{3,1}$ & $b_{3,2}$ & $b_{3,3}$ \\
\hline
\end{tabular}

Fig. 3 SubBytes function

\subsubsection{MixColumns function}

The MixColumns function's main aim is to transform the column encryption process, and its operation is a matrix multiplied by the state matrix to be a new matrix for each column in a repeat operation to get a new state of matrix data. The implementation of the MixColumns function is shown in Fig. 5.

Several rounds of the above function $(9,11,13)$ were executed with the possibility of encrypting data, to use such encrypted data for transfer; although it will cost a large computational cost, it can be used to secure data.

\section{Analysis of transmission characteristics of drilling fluid pulse signal}

4.1 The basic equation of information transmission

Drilling fluid pulses transmit signals in the form of pressure waves. Pressure wave is a kinetic energy and potential energy conversion process between. In the wellbore, the propagation of drilling fluid pulses can be treated as a one-dimensional unsteady flow. The basic equation describing the propagation characteristics of drilling fluid pulse can be obtained by applying the equation of motion of one-dimensional unsteady flow in the pipeline and closing the continuity Eq. 1.

$$
\left\{\begin{array}{l}
\frac{\partial V}{\partial t}+V \frac{\partial V}{\partial x}+g \frac{\partial H}{\partial x}+\frac{f V|V|}{2 D}=0 \\
\frac{\partial V}{\partial t}+V \frac{\partial V}{\partial x}+V \sin \alpha+\frac{\alpha^{2} \partial V}{g \partial x}=0
\end{array}\right.
$$

In the formula,
$H$-water head, m;

$f$-pipeline loss factor;

$V$-drilling fluid velocity, $\mathrm{m} / \mathrm{s}$;

$x$-pipeline axial coordinate;

$a$-pulse wave velocity of drilling fluid, $\mathrm{m} / \mathrm{s}$; and

$D$-drill stem diameter, $\mathrm{mm}$.

Drilling fluid channel is actually a multi-layer cylindrical waveguide, which consists of drill string drilling fluid, drill string, and annulus drilling fluid. The strata in the figure can be open-hole formations, casing and risers in offshore drilling. Multi-layer coupling models are complex and need to be appropriately simplified based on the issues studied.

(1) Do not consider the viscosity of the drilling fluid; (2) drilling fluid flow velocity relative to the pressure wave propagation speed is small, can be ignored; (3) wavelength of the pressure wave signal is much larger than the radius of the drill string; and (4) drill string axisymmetric homogeneous elastomer. Under the cylindrical coordinate system, the equation of motion of the multi-layer coupled model is shown in Eq. 2-3.

(1)Longitudinal vibration equation of the drill pipe

$E \frac{\partial^{2} u_{p}}{\partial z^{2}}=\rho_{p} \frac{\partial^{2} u_{p}}{\partial t^{2}}+\frac{2 v \rho_{i}}{A_{v}} \frac{\partial^{2} u_{i}}{\partial t^{2}}-\frac{2 v \rho_{i}\left(A_{i}+A_{p}\right)}{A_{p}} \frac{\partial^{2} u_{0}}{\partial t^{2}}$

(2)Longitudinal vibration equation of drilling fluid in drill string

\begin{tabular}{|l|l|l|l|}
\hline$a_{0,0}$ & $a_{0,1}$ & $a_{0,2}$ & $a_{0,3}$ \\
\hline$a_{1,0}$ & $a_{1,1}$ & $a_{1,2}$ & $a_{1,3}$ \\
\hline$a_{2,0}$ & $a_{2,1}$ & $a_{2,2}$ & $a_{2,3}$ \\
\hline$a_{3,0}$ & $a_{3,1}$ & $a_{3,2}$ & $a_{3,3}$ \\
\hline
\end{tabular}$\rightarrow$ ROL1 ROL2 $\rightarrow$\begin{tabular}{|l|l|l|l|}
$\mathrm{b}_{0,0}$ & $b_{0,1}$ & $b_{0,2}$ & $b_{0,3}$ \\
\hline$b_{1,0}$ & $b_{1,1}$ & $b_{1,2}$ & $b_{1,3}$ \\
\hline$b_{2,0}$ & $b_{2,1}$ & $b_{2,2}$ & $b_{2,3}$ \\
\hline$b_{3,0}$ & $b_{3,1}$ & $b_{3,2}$ & $b_{3,3}$ \\
\hline
\end{tabular}

Fig. 4 ShiftRows function 


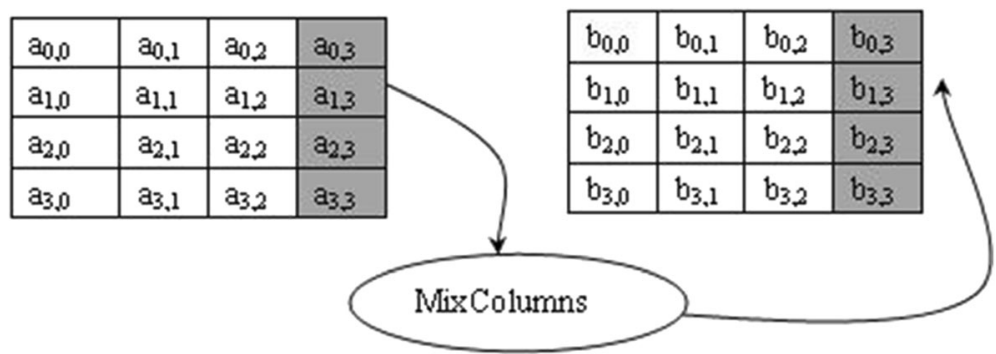

Fig. 5 MixColumns function

$$
\begin{gathered}
B_{i} \frac{\partial^{2} u_{i}}{\partial z^{2}}=2 v B_{i} \frac{\rho_{p}}{E} \frac{\partial^{2} u_{p}}{\partial t^{2}}+\left(1+\frac{2 B_{i}}{E}\left(\frac{2 A_{i}}{A_{p}}+1+v\right)\right) \\
p_{i} \frac{\partial^{2} u_{i}}{\partial t^{2}}-\frac{4 B_{0}}{E} \frac{A_{i}+A_{p}}{A_{p}} \rho_{0} \frac{\partial^{2} u_{0}}{\partial t^{2}}
\end{gathered}
$$

\subsection{Transmission speed influence}

Drilling fluid contains clay, cuttings, barite powder, and other solid substances, and often, there is a free state of the gas. Therefore, the drilling fluid flow is gas, liquid, solid three-phase flow. However, the solid particles in the drilling fluid are finer, suspended in the liquid, the concentration distribution is more uniform, and the gas content is usually small; so the gas, liquid, solid threephase flow rate is basically the same. The propagation velocity of the drilling fluid pulse depends on the elastic modulus of the system and the density of the fluid. According to the multi-phase flow theory, the density of the drilling fluid mixture and the bulk elastic modulus of the wellbore system can be expressed as Eq. 4-5.

$$
\begin{aligned}
& \rho=\left(1-\beta_{\mathrm{g}}-\beta_{\mathrm{s}}\right) \rho_{\mathrm{l}}+\beta_{\mathrm{g}} \rho_{\mathrm{g}}+\beta_{\mathrm{s}} \rho_{\mathrm{s}} \\
& \frac{1}{K_{e}}=\frac{1}{K_{\mathrm{l}}}\left[1+\psi \frac{K_{\mathrm{l}} D}{E_{e}}+\beta_{\mathrm{g}}\left(\frac{K_{\mathrm{l}}}{K_{\mathrm{g}}}-1\right)+\beta_{\mathrm{s}}\left(\frac{K_{\mathrm{l}}}{K_{\mathrm{s}}}-1\right)\right]
\end{aligned}
$$

In the formula,

$\rho_{\mathrm{g}}$-the density of the gas, $\mathrm{kg} / \mathrm{m}^{3}$;

$\rho_{\mathrm{l}}$-the density of the liquid, $\mathrm{kg} / \mathrm{m}^{3}$;

$\rho_{\mathrm{s}}$-the density of solid, $\mathrm{kg} / \mathrm{m}^{3}$;

$\beta_{\mathrm{g}}$-volumetric gas content, $\mathrm{m}^{3} / \mathrm{m}^{3}$;

$\beta_{\mathrm{s}}$-solid volume concentration, $\mathrm{m}^{3} / \mathrm{m}^{3}$;

$K_{\mathrm{g}}$-the volume elastic modulus of gas, Pa;

$K_{\mathrm{l}}$-the volume elastic modulus of liquid, $\mathrm{Pa}$;

$K_{\mathrm{s}}-$ the volume elastic modulus of solid, Pa;

$D$-pipe internal diameter, $\mathrm{m}$; and

$E$-elastic modulus of pipes, $\mathrm{Pa}$;

The factors that affect the transmission rate of drilling fluid pulse can be summarized as follows: composition and properties of drilling fluid, drill string size and material properties, and environmental parameters. Drilling fluid pulse transmission method is the main method of wireless information transmission in the drilling guidance parameter transmission. The transmission speed of drilling fluid pulse signal is an important point in the transmission characteristics of drilling fluid pulse signal, which is of great significance to the design of drilling guidance parameter transmission system, drilling fluid pulse signal transmission speed and drilling fluid temperature, drilling fluid composition and pulse pressure, and drilling fluid solid content and gas content, drill string diameter, and other factors. In the actual mining conditions, the drilling fluid is a kind of solid, liquid, and gas multi-phase mixed fluid. Drilling fluid contains solid material such as cuttings and contains gaseous substances such as air bubbles. The theoretical formula of pulse transmission speed of drilling fluid pulse generator is proposed. Considering the influence of drilling fluid temperature, drilling fluid composition, pulse pressure, drilling fluid solid content, gas content, diameter of drill string and other factors, the unsteady flow theory is used to deduce the pulse transmission velocity of drilling fluid pulse generator The theoretical formula is in the mud pulse transmission system; the signal transmission speed is a basic parameter. However, drilling fluids contain solids such as clays, cuttings, barites, and the like, and the presence of free-standing gases tends to form bubbles, increasing the complexity of the signaling speed problems.

\subsection{Pulse downward system model analysis}

The circulation channel of the system drilling fluid can be divided into two parts: the circulation channel and the discharge channel. When the surface discharge device is closed, the drilling fluid will circulate normally along the circulation channel. When the surface discharge device is opened, the drilling fluid is divided along the circulation channel flow, part of the drilling fluid along the discharge channel will return directly to the mud pool, and then complete the drilling fluid pulse transmission [23, 24]. Wellbore trajectory control 


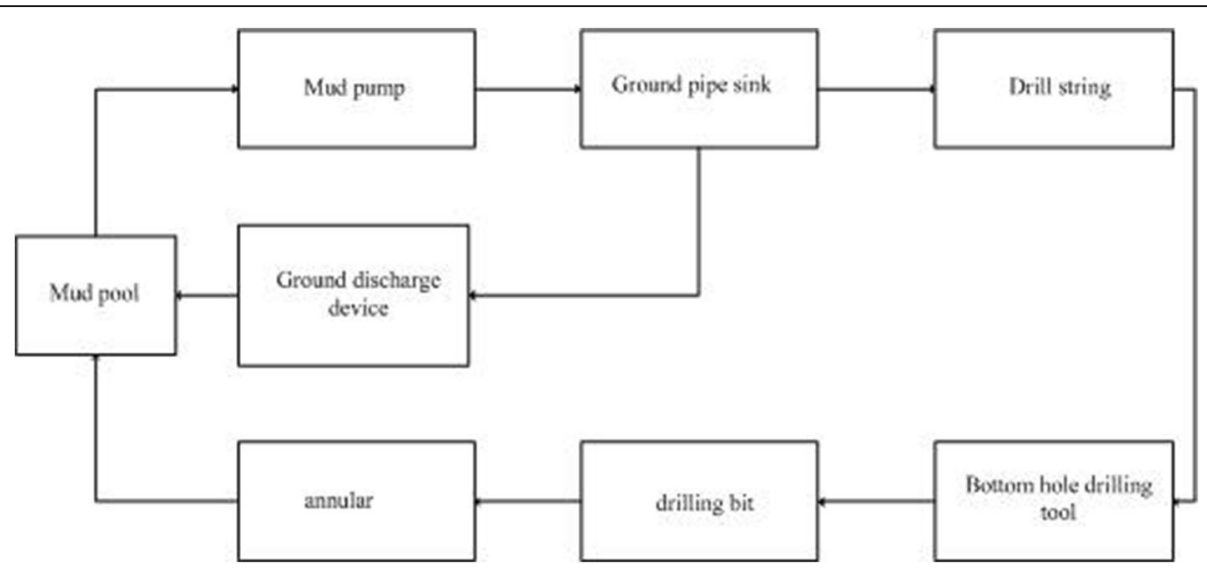

Fig. 6 Drilling fluid circulation system block diagram

drilling fluid circulation system can be simplified as shown in Fig. 3 block diagram. As shown in Fig. 6, the pressure loss of the drilling fluid flowing in the main circulation loop is mainly composed of flow, drill bit, and annulus flow.

The negative pulse command transfer is realized by switching the open and close of the current limiting valve. Therefore, the interference signal generated during the transmission is the disturbance of the frequency of other influencing factors: the frequency disturbance generated by the piston pump, the bottom hole power machine, the influence of bubbles in the slurry on the signal transmission frequency, the frequency disturbance caused by the moving drill, The interference frequency and signal transmission frequency of the larger difference can be filtered out by the filter.

\section{Conclusions}

In the simulation of drilling rig site test, the signal receiving short section is adjusted and connected between the two drill collars of the near bit and into the well with the drilling tool. One end of the ground information, downloading device is connected with the three-way connecting pipe of the high-pressure riser pipe, and the other end is connected with the mud tank back-flow port. The flow valve opens to a pre-designed opening to open the shut-off valve between the standpipe and the information drop unit. When the signal reception short reaches the underground predetermined depth, the mud pump is turned on to establish the circulation condition. After the mud pump is stable, save the pump pressure to maintain a certain pump pressure, and then, use the computer control information to realize the different time intervals, the preset pressure pulse signal is sent out. The signal receiving short section receives the pulse signal from the ground pressure in the well bottom and stores it. According to the test results, it can be seen that the increase of the flow valve opening and the increase of the pump pressure have obvious improvement on the information transmission. The deeper the depth of field, the greater the impact on information delivery. The width of the pulse width increases; the information has significantly improved, especially in the depth of the case; but more than a certain value of the effect is not obvious, the pulse width interval value of $9 \mathrm{~s}$ or so; to improve the information pass to take the signal suppression approach, that is, to increase the signal gap on the ground, so that the original received useful signal is much larger than the noise. In the process of information transmission, the depth, pump pressure, pulse width, and flow valve open four factors together, because the depth and pump pressure by the engineering conditions, so the quantitative flow valve opening and pulse width interval is the important factors that should be identified before work.

\section{About the authors}

Lin DeShu received his M.S. degree in Pattern Recognition and Intelligent System from Guilin University of Electronic Technology in Guilin, China. He is a doctoral student in the oil field equipment at Yangtze University. His research interest is mainly in the area of Computer Software, Mechanical and Electrical Integration. He has published several research papers in scholarly journals in the above research areas and has participated in several books. Feng Ding received his Ph.D. degree in mechanical design and theory from China University of Petroleum in Beijing, China. He is currently a professor in the School of Mechanical Engineering at Yangtze University. His research interest is mainly in the area of the petroleum down hole tools, rotary guide system design, and offshore oil down hole tool. He has published one hundred and fifty papers in scholarly journals in the above research areas and has participated in several books.

\section{Authors' contributions}

FD is the corresponding author of the paper. FD is responsible for all aspects of the paper. LDS is the first author of the paper. LDS contributed the idea of the paper. Both authors read and approved the final manuscript.

\section{Competing interests}

The authors declare that they have no competing interests.

\section{Publisher's Note}

Springer Nature remains neutral with regard to jurisdictional claims in published maps and institutional affiliations. 


\section{Author details}

'School of Computer Science, Yangtze University, Jingzhou, Hubei 434023,

China. ${ }^{2}$ School of Mechanical Engineering, Yangtze University, Jingzhou,

Hubei 434023, China.

Received: 17 October 2017 Accepted: 13 December 2017

Published online: 21 December 2017

\section{References}

1. Mougel B, et al. Apparatus and method for communication with downhole equipment using drill string rotation and gyroscopic sensors. United states: 6,267. 185. 2001.

2. Engelder PD. Method and apparatus for telemetry while drilling by changing drill string rotation angel or speed. United states patent: 4763258. 1998.

3. Apparatus and method for self adjusting downlink signal communication. United States Patent:5,963,138. 1999.

4. Finke, et al. Downlink telemetry system. United States Patent:6,920,085. 2005

5. MORIARTY KEITH A(US). Rotary downlink system. UK Patent Application: 241212821.09. 2005.

6. Treviranus J, et al. Method and apparatus for downlink communication. United States Patent Application. 2006.

7. Herrmaun, et al. Communication with a downhole tool. United States Patent:6,550,538. 2003.

8. Yinao, S, \& Xiushan, L (2002). Propagation characteristics of mud pulse signal in wellbore, Key Laboratory of Drilling Engineering, China National Petroleum Corporation. Underground engineering technology symposium proceedings (pp. 86-94). Beijing: Petroleum Industry Press.

9. Xiushan, L, \& Yinao, S. (2000). Study on transmission velocity of mud pulse signal. Petrol. Drill. Tech., (05), 24-26.

10. Xiu-Shan, L, \& Jun, G. (2000). Study on the transmission velocity distribution of drilling fluid pulse, Drilling \& Production Technology, 23(4), 74-76.

11. Xiusan, L, Yi'nao, S, Zhangzhi, C. (1999). Analysis of influence factors on drilling mud pulse transmission speed. Drill. Prod. Technol., 21(5), I-4.

12. Xiu-shan, LIU, \& Yi-nao, S. (2000). Scheme design of downward signaling system. Acta Petrol. Sin., 21(6), 88-92.

13. Wei, Z, \& Wang, X (2002). Geostationary information transmission channel. In Key Laboratory of Drilling Engineering, China National Petroleum Corporation. Underground Engineering Technology Symposium, (pp. 44-49). Beijing: Petroleum Industry Press.

14. Lin, L, Limin, S, Xiaoli, Z (2002). Research on short distance wireless transmission of underground data. In Key Laboratory of Drilling Engineering, China National Petroleum Corporation. Underground Engineering Technology Symposium, (pp. 64-68). Beijing: Petroleum Industry Press.

15. Haiyan, S, Jing, Z, XinSheng, F. (1999). JYGJ-1 instrument for information transmission from surface to downhole. J. Xi'an Petrol. Inst., 14(1), 30-32.

16. Jing, Z, Haiyan, S, Changxing, L. (1999). Decoding of the communication channel from down hole to surface in drilling well. J. Xian Petrol. Inst, 14(2), 2 7 31.

17. Haiyan, S, Jing, Z, Gang, F. (2002). A method of realizing the communication from ground to downhole in closed-loop drilling. J. Xi'an Petrol. Inst. (Natural Science Edition), 17(2), 70-73.

18. Haiyan, S, Jing, Z, XinSheng, F. (2003). Downlink realized by the mud-pulse width modulation. Petrol. Instrument, 17(1), 10-12.

19. Yang, Q, \& Jin, L. (2004). A scheme for downward communication in the steerable rotary directional drilling system. Petrol. Geology Recov. Efficiency, 11(1), 75-77.

20. Abdallah $\mathrm{AH}$, et a1. Analysis and identification of a drilling fluid column based on decoding of measurement-while-drilling signals. United States Patent:5,055,83. 1991

21. Jeffryes $B P$, et a1.Signal recognition system for wellbore telemetry. United States Patent:5,955,966. 1999.

22. Xiushan, L, \& Yinao, S. (2000). Study on transmission velocity of mud pulse signal. Petrol. Drill. Tech., 28(5), 24-26.

23. Xiushan, L, \& Yinao, S. (2000)). Investigation on the transmission behaviors of drilling fluid pulse signal. Oil Drill. Product. Technol., (04), 8-10+83.

24. Fengfei, L, Shiquan, J, Hanxing, L, Gao, D. (2012)). Study on downward signaling system of rotarysteerable drilling tool. Chin. Offshore Oil Gas, (06), 42-46+65.

\section{Submit your manuscript to a SpringerOpen ${ }^{\circ}$ journal and benefit from:}

- Convenient online submission

- Rigorous peer review

- Open access: articles freely available online

- High visibility within the field

Retaining the copyright to your article

Submit your next manuscript at $>$ springeropen.com 